\title{
A Legendre Polynomial Integral
}

\author{
By James L. Blue
}

Abstract. Let $\left\{P_{n}(x)\right\}$ be the usual Legendre polynomials. The following integral is apparently new.

$$
\int_{0}^{1} P_{n}(2 x-1) \log \frac{1}{x} d x=\frac{(-1)^{n}}{n(n+1)} \text { for } n \geq 1 .
$$

It has an application in the construction of Gauss quadrature formulas on $(0,1)$ with weight function $\log (1 / x)$.

1. Motivation. For integrals of the type $\int_{a}^{b} f(x) w(x) d x$, where $w(x)$ is positive in $(a, b)$, Gaussian quadrature formulas of the type

$$
\int_{a}^{b} f(x) w(x) d x \approx \sum_{k=1}^{n} h_{k n} f\left(\xi_{k n}\right)
$$

are often useful. The $\left\{h_{k n}\right\}$ and $\left\{\xi_{k n}\right\}$ are chosen to make the formulas exact when $f(x)$ is a polynomial of degree $2 n-1$ or less [1]. These formulas are especially useful when $w(x)$ is singular at one or more points in the interval.

The method of modified moments [2], [3] , [4] provides a stable method for calculating the $\left\{h_{k n}, \xi_{k n}\right\}$ if the set of polynomials orthogonal on $(a, b)$ with weight function $w(x)$ are known. That is, a set of $\left\{Q_{k}\right\}$, such that

$$
\int_{a}^{b} Q_{k}(x) Q_{m}(x) w(x) d x=0 \text { if } k \neq m
$$

is desired. Any such family of orthogonal polynomials obeys a three-term recurrence relation [5],

$$
\begin{aligned}
& Q_{-1}(x)=0, \quad Q_{0}(x)=1, \\
& x Q_{k}(x)=a_{k} Q_{k+1}(x)+b_{k} Q_{k}(x)+c_{k} Q_{k-1}(x), \quad k \geqslant 1,
\end{aligned}
$$

with $a_{k} \neq 0$.

For some intervals and weight functions, the orthogonal polynomials are known, and there is no problem. For example, if $a=-1, b=+1$, and $w(x)=1$, the usual Legendre polynomials $\left\{P_{k}(x)\right\}$ are an orthogonal set,

$$
\int_{-1}^{1} P_{k}(x) P_{m}(x) d x=0 \text { if } k \neq m .
$$

For most intervals and weight functions, the corresponding orthogonal polynomials are not known. If the moments $\int_{a}^{b} x^{k} w(x) d x$ are known, the $\left\{a_{k}, b_{k}, c_{k}\right\}$ of the unknown set of orthogonal polynomials can be found [2], but the process is nu-

Received February 24, 1978.

AMS (MOS) subject classifications (1970). Primary 65D30. 
merically unstable [3], [4]. More generally, if $\left\{\bar{Q}_{k}\right\}$ is any set of polynomials, not necessarily obeying any orthogonality relation, but obeying a three-term recurrence relation

$$
x \bar{Q}_{k}(x)=\bar{a}_{k} \bar{Q}_{k+1}(x)+\bar{b}_{k} \bar{Q}_{k}(x)+\bar{c}_{k} \bar{Q}_{k-1}(x),
$$

the $\left\{a_{k}, b_{k}, c_{k}\right\}$ of the unknown set of orthogonal polynomials can be found [4]. For this, the modified moments $\int_{a}^{b} \bar{Q}_{k}(x) w(x) d x$ are needed. The stability of the process depends on the $\left\{\bar{Q}_{k}\right\}$. Some particular examples [3], [4] suggest that, for finite $a$ and $b$, the process is probably stable if the $\left\{\bar{Q}_{k}\right\}$ are themselves orthogonal polynomials with some weight function $\bar{w}(x)$.

The appropriate orthogonal polynomials for

$$
\int_{0}^{1} f(x) \log \frac{1}{x} d x
$$

are not known analytically. The Altran symbolic algebra package [6] was used to calculate the modified moments for various sets of orthogonal polynomials. The shifted Legendre polynomials [5], $\left\{P_{k}^{*}(x)\right\}$, with $P_{k}^{*}(x)=P_{k}(2 x-1)$, were found to have a particularly simple formula for modified moments, and the algorithm of [4] was found to be stable.

\section{A Legendre Polynomial Integral.}

THEOREM. Let $P_{n}^{*}(x)$ be the nth shifted Legendre polynomial. Define $\nu_{n}=$ $\int_{0}^{1} P_{n}^{*}(x) \log (1 / x) d x$. For $n \geqslant 1, \nu_{n}=(-1)^{n} / n(n+1)$.

Proof. By induction. Using $P_{k}^{*}(x)=P_{k}(2 x-1)$, from [5] we obtain

$$
\begin{gathered}
P_{0}^{*}(x)=1, \quad P_{1}^{*}(x)=2 x-1, \quad P_{2}^{*}(x)=6 x^{2}-6 x+1, \\
(k+1) P_{k+1}^{*}(x)=(2 k+1)(2 x-1) P_{k}^{*}(x)-k P_{k-1}^{*}(x), \quad k \geqslant 2 .
\end{gathered}
$$

Note that $P_{n}^{*}(1)=1$. The first three modified moments are $\nu_{0}=1, \nu_{1}=-1 / 2$ and $v_{2}=1 / 6$. We define $\mu_{n}=\int_{0}^{1}(2 x-1) P_{n}^{*}(x) \log (1 / x) d x$.

Assume $\nu_{k}=(-1)^{k} / k(k+1)$ for $k \geqslant 2$. Using the recurrence relation,

$$
\nu_{k+1}=\int_{0}^{1} P_{k+1}^{*}(x) \log \frac{1}{x} d x=\frac{1}{k+1}\left[(2 n+1) \mu_{k}-k \nu_{k-1}\right] .
$$

Also from [5], the derivative of $P_{k}^{*}(x)$ is

$$
\frac{d}{d x} P_{k}^{*}(x)=\frac{-k}{2 x(1-x)}\left[(2 x-1) P_{k}^{*}(x)-P_{k-1}^{*}(x)\right]
$$

Integrate by parts in the definition of $\mu_{k}$ to obtain

$$
\begin{aligned}
\mu_{k}= & \left.P_{k}^{*}(x)\left[x(1-x) \ln x+\frac{1}{2} x^{2}-x\right]\right|_{0} ^{1} \\
& +\frac{k}{4} \int_{0}^{1} \frac{x-2}{1-x}\left[(2 x-1) P_{k}^{*}(x)-P_{k-1}^{*}(x)\right] d x . \\
& -\frac{k}{2} \int_{0}^{1}\left[(2 x-1) P_{k}^{*}(x)-P_{k-1}^{*}(x)\right] \log \frac{1}{x} d x
\end{aligned}
$$

Simplifying, and using $P_{k}^{*}(1)=1$, 


$$
\mu_{k}=-\frac{1}{2}-\frac{k}{2} \mu_{k}+\frac{k}{2} \nu_{k-1}-\frac{1}{2} \int_{0}^{1} x(x-2)\left[\frac{d}{d x} P_{k}^{*}(x)\right] d x
$$

The last integral may be integrated by parts, giving

$$
-\left.\frac{1}{2} x(x-2) P_{k}^{*}(x)\right|_{0} ^{1}+2 \int_{0}^{1}(x-1) P_{k}^{*}(x) d x .
$$

The integrated term is $1 / 2$, and the integral is zero for $k>1$ because of the orthogonality of the $\left\{P_{\boldsymbol{k}}^{*}\right\}$. Thus,

$$
\mu_{k}=\frac{k}{2}\left(\nu_{k-1}-\mu_{k}\right), \quad \mu_{k}=\frac{k}{k+2} \nu_{k-1} .
$$

Inserting this result in (1),

$$
\begin{aligned}
\nu_{k+1} & =\frac{k}{k+1}\left[\frac{2 k+1}{k+2}-1\right] \nu_{k-1}=\frac{k(k-1)}{(k+1)(k+2)} \frac{(-1)^{k-1}}{k(k-1)} \\
& =\frac{(-1)^{k+1}}{(k+1)(k+2)}
\end{aligned}
$$

Bell Laboratories

Murray Hill, New Jersey 07974

1. P. J. DAVIS \& P. RABINOWITZ, Numerical Integration, Blaisdell, Waltham, Mass., 1967.

2. G. H. GOLUB \& J. H. WELSCH, "Calculation of Gauss quadrature rules," Math. Comp., v. 23,1969 , pp. $221-230$.

3. W. GAUTSCHI, "On the construction of Gaussian quadrature rules from modified moments," Math. Comp., v. 24, 1970, pp. 245-260.

4. R. A. SACK \& A. F. DONOVAN, "An algorithm for Gaussian quadrature given modified moments," Numer. Math., v. 18, 1972, pp. 465-478.

5. U. HOCHSTRASSER, "Orthogonal polynomials," in M. Abramowitz and I. A. Stegun (eds.), Handbook of Mathematical Functions, Dover, New York, 1965.

6. W. S. BROWN, Altran User's Manual, 4th ed., Bell Laboratories, Murray Hill, N. J., 1977. 